Die von Warburg beschriebene Aktivierung der photochemischen Chinonreduktion der Granula in vitro durch Chlorionen (beste Wirkung bei $m / 150-\mathrm{KCl}$ ) legt die Annahme einer entsprechenden Chloridwirkung auch bei der Photosynthese in vivo nahe. Eine Notwendigkeit des Chlorids haben wir bei der Algenkultur unter unseren Anzuchtbedingungen nicht beobachten können. Mit der Wirksamkeit schwer entfernbarer Spuren von Chlorid muß allerdings gerechnet werden. Man könnte auch vermuten, daß die von Warburg benutzten Granula aus Chenopodiaceen (Spinat, Zuckerrübe) eine spezifische Strukturanpassung an deren relativ hohen Chloridgehalt aufweisen. Übrigens fand Warburg, daß auch Nitrationen eine Aktivierung der Chinonreduktion durch die Granula herbeiführen können, wenn auch mit geringerer Wirksamkeit als die Chlorionen.

Eine völlige Reversibilität der Photosynthesehemmung bei Mineralsalzmangel haben wir nicht mehr beobachten können, wenn einmal der Chlorophyllgehalt der Mangelzellen erheblich vermindert war. Für die im Erholungsverlauf erreichbare photosynthetische Maximalleistung im Starklicht scheint dann der Chlorophyllgehalt begrenzender Faktor zu sein. Wahrscheinlich handelt es sich aber hier nur um eine vorgetäuschte Abhängigkeit. Denn unter den tiefergreifenden Mangelbedingungen dürften Veränderungen in der Plasmazusammensetzung (z. B. im Gehalt an Chloroplasteneiweiß) eintreten, welche ihrerseits im Starklicht die Photosynthese mehr und mehr irreversibel stillegen. Dabei kann der Chlorophyllgehalt den Indikator solcher Veränderungen bilden, ohne selbst für die Photosynthesehemmung unmittelbar maßgeblich zu sein.

S poehr und Milner ${ }^{14}$ haben gezeigt, daß unter der Wirkung von Außenfaktoren, insbesondere auch der Mineralsalzernährung, der Lipoid- bzw. Fettgehalt von Algen außerordentlich variieren kann. Von dem Algenmaterial unserer Versuche enthielten die N-Mangelzellen mikroskopisch sichtbare Fetttröpfchen, während die Kalimangelzellen frei davon waren. Es fällt daher auf, daß im kurzfristigen Versuch keine ausgeprägten Gaswechselabweichungen infolge Fettbildung bemerkbar werden. My er s ${ }^{7}$ hat entsprechende Erfahrungen gemacht. Immerhin sei darauf hingewiesen, daß die fettfreien bzw. -armen Kalimangelzellen im Starklicht einen P. Q. von genau - 1 besitzen, die N-Mangelzellen dagegen, bei denen eine Nitratverarbeitung in merklichem Ausmaß nicht in Betracht kommt, einen gleichbleibenden Überschuß der $\mathrm{O}_{2}$-Abgabe gegenüber der $\mathrm{CO}_{2}$-Aufnahme zeigen (P.Q. $=-1,05)$; hierbei dürfte es sich um Extrakohlensäureausscheidung im Zusammenhang mit einem zur Fettbildung führenden Umbau von Endassimilaten oder Photosynthese-Zwischenprodukten handeln. Es bleibt zu prüfen, ob unter anderen Anzuchtbedingungen (z. B. höherer Lichtintensität) oder mit anderen Algenstämmen eine höhere Fettproduktion sich durch sțärkere Abweichungen des Gaswechsels deutlicher bemerkbar macht.

Eine ausführlichere Darstellung der hier z. Tl. nur kurz wiedergegebenen Befunde wird an anderer Stelle erfolgen.

\title{
Über das Vorkommen von zweierlei Fasern in der Muskulatur von Haien
}

\author{
Von PaUl Krüger \\ Aus dem Zoologischen Institut der Universität Heidelberg \\ (Z. Naturforschg. 5 b, 218-220 [1950]; eingegangen am 8. Mai 1950)
}

\begin{abstract}
Im Schwanz der Haie findet sich um die Wirbelsäule liegend eine stark durchblutete Schicht von dünnen, sarkoplasmareichen Fasern mit „Felderstruktur“, mit wenigen randständigen Kernen. Die mächtige äußere „weiße“ Muskelschicht enthält ebenso ausschließlich dicke, relativ sarkoplasmaarme Fasern mit „Fibrillenstruktur“ mit zahlreichen Kernen.
\end{abstract}

$\mathrm{D}$ er Nachweis, daß es in den Muskeln der Anuren zwei charakteristisch verschiedene Arten von Muskelfasern gibt ${ }^{1}$, deren chemische Beschaffenheit und reizphysiologisches Verhalten nur mit den beiden Leistungen der Muskeln: Bewegen und Halten (Tetanus und Tonus), in Beziehung zu setzen sind ${ }^{2}$ — sie besitzen je auch eine spezif. Innervation ${ }^{3,4}$,

1 P. K r ü g e r, Biol. Zbl. 49 [1929]; F. F ü r l in g e r, Zool. Anz. 90 [1930]. brachte die weitere Verpflichtung, auch in den Skelettmuskeln der übrigen Wirbeltiergruppen Vorkommen und Verteilung dieser Fasern in Muskeln bestimmter Funktion aufzudecken. Wir haben diese Aufgabe

2 P. Kr üger u. F. Fürlinger, Ak. Anz. Akad. Wiss. Wien, math.-naturwiss. Kl. 1931. - P. K r üg e r, F. Duspiva u. F. F ürlinger, Pflügers Arch. ges. Physiol. Menschen Tiere 231 [1933].

3 P. Gg. G ü n th e r, Anat. Anz. 97 [1950].

4 P. K r ü g e r, Zool. Anz. (im Druck). 
prinzipiell gelöst (Urodelen ${ }^{5}$, Reptilien ${ }^{6}$, Aves ${ }^{7,4}$ und Mammalia ${ }^{8}$ ). Es gelang uns (G ü $n$ the $r^{9}$ und mir), beide Faserarten auch in menschlichen Muskeln festzustellen (unveröff.). Die Übereinstimmung unserer, an Fröschen gewonnenen Ergebnisse experimenteller Untersuchungen, mit denen zahlreicher anderer Autoren an Anuren, Vögeln und Säugetieren gab zum erstenmal die Möglichkeit, eine einheitliche Auffassung über die Grundlagen und das Wesen des Tetanus und Tonus der quergestreiften Skelettmuskeln der Wirbeltiere zu formulieren ${ }^{10}$. Dabei ist die Beschränkung auf die quergestreifte Skelettmuskulatur der Tetrapoden, die ich uns auferlegt hatte, nur von Vorteil gewesen. Aus verschiedenen - in der Materie gelegenen - Gründen habe ich zunächst auch die Zungen- und Augenmuskeln beiseite gelassen.

Unsere Untersuchungen - wie aber auch die Ergebnisse anderer Autoren - zeigten, daß die Farbe der Muskeln (weiß, hell oder rot) nicht ursächlich mit der Leistung eines Muskels verknüpft ist. „Rot“ ist nicht eine Eigenschaft tonischer Muskeln oder das Anzeigen für eine tonische Funktion. Die meisten rein tetanischen Muskeln der tetrapoden Wirbeltiere sind rot! Diese Farbunterschiede treten vor allem bei domestizierten Tieren (Vögeln und Säugetieren), bei denen sie auch zuerst wissenschaftliches Interesse gefunden haben (L or enzini, 1678), in Erscheinung. Welche Bedeutung dem Myohämoglobin jedoch zukommt, ist auch heute noch nicht restlos geklärt.

Die von uns aufgefundenen Fasern mit „Felderstruktur" (irrtümlich früher von uns als "Säulchenfelderung " nach S ch aff e r ${ }^{11}$ bezeichnet) sind auch nicht unmittelbar gleichbedeutend mit sarkoplasmareichen Fasern, wie sie $\mathrm{Rollet \textrm {t } ^ { 1 2 }}$ als erster bei Teleostiern beschrieben hat, und wie sie $\mathrm{Knoll}^{13}$ als charakteristische Faserart bei allen Tiergruppen - Wirbeltiere und Wirbellose - ansah, wobei er aber infolge unzureichender Technik (Goldfärbung; s. Kritik durch v. Ebner ${ }^{14}$; vgl. Romei ${ }^{15}$ ) zu vielfach irrtümlichen Beobachtungen kam. Infolge-

5 H. Th i e l, Z. Zellforsch. 30 [1939].

6 H, K ö h l e r, Z. Zellforsch. 28 [1938].

7 J. Th. Nel, Verh. naturhist.-med. Ver. Heidelberg, N. F. 18 [1940].

8 F. D a n z i nge r, Z. Zellforsch. 25 [1936].

9 P. Gg. Günth er, Med. Klinik Mainz (Vorstand Prof. Dr. Voit).

10 P. K r üg er, Anat. Anz. 97 [1950]; Experientia 6 [1950].

11 J. S ch a ff er, S.-B. Akad. Wiss. Wien, math.-naturwiss. Kl. Abt. III, 102 [1893].

12 A. Rollett, Arch. mikr. Anat. 32 [1888]. dessen sind auch seine zahlreichen Tafelabbildungen fast gänzlich unbrauchbar.

Noch andere Unterschiede zwischen den Muskelfasern hatte man als charakteristisch für schnelle (tetanische) oder langsame (tonische) Leistung angesehen. Das waren u. a. der Faserdurchmesser (dicke oder dünne Fasern) und Zahl und Lage der Kerne im Muskelfaserquerschnitt.

Es muß aber betont werden, daß bei den Tetrapoden alle diese angeblichen Gegensätze sich als fließende Größen oder nur für einzelne Tiere zutreffend erwiesen. Zur eindeutigen Charakterisierung von Fasern mit tetanischer oder tonischer Funktion können sie nicht verwendet werden.

In neuerer Zeit sind schließlich eine Reihe von Untersuchungen angestellt worden, die sich auf die Versorgung der Muskeln mit Blutgefäßen beziehen. Schon Ranvier ${ }^{16}$ hatte festgestellt, daß die Gestalt der Kapillaren und ihre mengenmäßige Verteilung im roten Muskel (Kaninchen, vor allem M. semitendinosus verwendet, der tatsächlich auch ein rein tonischer Muskel ist) abweicht vom gewohnten Verhalten, wie es der weiße Muskel (verwendet vor allem die $\mathrm{Mm}$. vastus internus und adductor magnus, die beide rein tetanische Muskeln sind) zeigt. Die Ergebnisse der neueren Arbeiten ${ }^{17}$ widersprechen ein. ander. Es scheint sich aber doch die Beobachtung von Ranvier zu bestätigen, daß Muskeln, die Dauerleistungen vollbringen - was nicht identisch mit einer tonischen Funktion ist, womit es oft zusammengeworfen worden ist! -, zahlreichere und anders geartete Kapillaren besitzen, wie solche, die nur zu kurzen Bewegungen verwendet werden (C a meron ${ }^{18}$; Kow es chnikowa ${ }^{17}$ ).

Bei der Bedeutung, die das Vorkommen von zwei verschiedenen Muskelfasern für die allgemeine und vergleichende Physiologie, wie besonders aber auch für die Klinik ${ }^{19}$ hat, ist es mehr als bemerkenswert, daß es Muskeln gibt, bei denen alle oben genannten

13 Ph. Knoll, Denkschr. Akad. Wiss. Wien, math.naturwiss. Kl. 58 [1891].

14 V. E b n e r, S.-B. Akad. Wiss. Wien, math.-naturwiss. Kl. Abt. III 129 [1920].

15 B. R o m e i s, Mikroskop. Technik, 15. Aufl., Leibniz-Verlag München 1948.

16 L. Ranvier, Arch. physiol. norm. pathol. Paris (2) 1 [1874].

17 G. S t o ël, Z. Zellforsch. 3 [1926]; J. W. D u y f f u. H. D. B o u m a n, Z. Zellforsch. 5 [1927]; A. K. Ko wes chnikowa, Bull. Inst. scient. Lesshaft 20 [1936]; W. S t e n d e l, Zool. Jb. Anat. Ont. 65 [1938].

18 M. L. Ca m e ro n, J. Physiology 67 [1929].

19 P. K r üg e r, Klin. Wschr. (im Druck). 
Differenzen in extremer Ausbildung zusammen vorkommen. Es handelt sich um Muskeln bei Haifischen.

Zunächst sei noch kurz berichtet, daß die Teleostier etwas abweichende Muskelfaserstrukturen zeigen, wie diese Tiergruppe ja auch sonst eine große Zahl von Sonderheiten aufweist. $\mathrm{Da} \beta$ es bei ihren Muskelfasern sich aber im Prinzip um die gleichen Unterschiede zwischen zwei Typen handelt, geht aus den Untersuchungen von $\mathrm{B}$ ü h n 20 und vor allem von $\mathrm{M}$ a s e r ${ }^{21}$, wie auch aus denen von $\mathrm{K}$ i r s c he 22 über deren Innervation hervor. (Seine Schlußfolgerungen über die Funktion der Seitenlinienmuskeln sind aber sicher nicht zutreffend 23.)

Vor Jahren beobachtete ich an einer Scheibe aus dem Schwanz eines Eishaies (Laemargus borealis), die in einer Fischhandlung gekauft worden war, daß die um die Wirbelsäule liegenden Muskelfasern sich durch eine blaurötliche Farbe von der sehr viel dickeren, hellen äußeren Schicht scharf begrenzt abhoben (vgl. $\left.{ }^{13}\right)$. Im zubereiteten Zustand (gebacken) unterschieden sie sich durch einen deutlichen Fischgeschmack (Trimethylamin) von dem weißen Fleisch ${ }^{24}$. Ende 1944 erhielt ich dann durch die Freundlichkeit von Hrn. Dr. A. Wulff, Hamburg, Stücke aus dem Schwanz eines Heringshaies (Lamna cornubica), die sich als gut fixiert (Susa) erwiesen. Nach Mitteilung von Dr. Wulff sind die Teile unmittelbar um die Wirbelsäule „sehr blutdurchsetzt“. Die histologische Untersuchung ergab folgendes.

Querschnitte durch Fasern aus beiden Zonen lassen schon bei schwacher Vergrößerung (Abb. $1^{*}$ ) - besonders eindrucksvoll bei Betrachtung des Randgebietes beider Fasern - erkennen, daß jede Zone einheitlich aus einer bestimmten Faserart aufgebaut ist. Das „weiße“ Fleisch der Randschicht enthält nur Fasern von großem Querschnitt, der den der Fasern der „roten“ Zone um etwa das 5-fache an Fläche übertrifft.

Was weiter sofort in die Augen fällt, sind Zahl und Lage der Kerne in den Fasern. Die dicken Fasern zeigen durchweg eine große Anzahl (16-18, meist

20 W. B üh n, Z. Zellforsch. 30 [1940].

21 J. M a s'e r, Zool. Jb. Anat. Ont. 70 [1949].

22 W. Kir s che, Anat. Anz. 96 [1948].

23 Vgl. Ber. wiss. Biol. 67, 160 [1950]. um 20), die über den ganzen Faserquerschnitt verteilt sind. Demgegenüber finden sich in den Querschnitten der dünnen Fasern nur wenige (0 bis höchstens 4), stets randständige Kerne. Auch die Längsschnitte lassen diesen Unterschied mit aller Deutlichkeit erkennen.

Verwendet man starke optische Systeme, so beobachtet man den wesentlichsten Unterschied zwischen beiden Faserarten. Die dicken sind durch eine typische, sehr feine „Fibrillenstruktur“ charakterisiert (Abb. 2), während die dünnen Fasern der roten Innenzone ausschließlich eine, mit den Zuständen bei den Tetrapoden - also abweichend von den Teleostiern! — übereinstimmende „Felderstruktur“ zeigen (Abb. 3 u. 4). Unter dem Sarkolemm befindet sich in diesen eine mehr oder weniger breite, z. Tl. wulstartig vorgewölbte Sarkoplasmaschicht, in der die Kerne liegen. Es sind also "sarkoplasmareiche“ Muskelfasern!, und die „Felder“ haben unmittelbar nichts mit der Unterscheidung in sarkoplasmaarme und sarkoplasmareiche Fasern zu tun! Im Vergleich zu den Tetrapoden sind bei den Haien auch die Fasern mit „Fibrillenstruktur“ sarkoplasmareich! Das zeigen flache Anschnitte oder Schrägschnitte durch die Faserenden (?). Während in den Muskeln der Tetrapoden die Zwischenräume zwischen den Fibrillen optisch leer sind - so ist es nach B üh n ${ }^{20}$ auch in den „primären Fasern“ (vgl. B ühn, Abb. 3) der Teleostier -, enthalten sie in den Fasern der Haie auch im fixierten Zustand Sarkoplasma. In den „Feldern" lassen sich vielfach mehrere Fibrillen erkennen.

Schließlich unterscheiden sich beide Zonen des Haifischschwanzes noch durch ihren Gehalt an Kapillaren. Ihre Zahl ist zwischen den dünnen sarkoplasmareichen Fasern mit „Felderstruktur“ auffallend größer als zwischen den dicken, relativ sarkoplasmaarmen Fasern mit „Fibrillenstruktur“ der Randzone.

24 Nach Hop pe-S e y le r (Verh. physik.-med. Ges. Würzburg, 1928) übertreffen Haifischmuskeln solche von anderen Seefischen im Gehalt an Trimethylaminoxyd, Ammoniak und Trimethylamin. 